\title{
Relativistic Alpha Field Theory - Part I: Determination of Field Parameters
}

\author{
Branko M. Novakovic
}

\begin{abstract}
General Relativity Theory (GRT) cannot be applied to the extremely strong gravitational field at the Planck's scale, because of the related singularity. Here we show that Relativistic Alpha Field (RAF) theory extends the application of GRT to the extremely strong fields at the Planck's scale. This is the consequence of the following predictions of RAF theory: a) no a singularity at the Schwarzschild radius, b) there exists a minimal radius at $\mathbf{r}=\left(\mathrm{GM} / 2 \mathrm{c}^{2}\right)$ that prevents singularity at $\mathbf{r}=$ 0 , i.e. the nature protects itself, c) the gravitational force becomes positive (repulsive) if $\left(\mathrm{GM} / \mathrm{rc}^{2}\right)>1$, that could be a source of a dark energy, and d) unification of electrical and gravitational forces can be done in the standard four dimensions (4D). Predictions a) and b) are presented in the second part of this theory, while predictions c) and d) are considered in the third part of the theory. In this (first) part of the theory we present the solution of the field parameters in RAF theory. This solution is based on the assumption that the field parameters should connect geometry of the line element with potential energy of a particle in an alpha field. The solution of the field parameters is the key point in this theory. If RAF theory is correct, then it could be applied to the both weak and strong fields at the Universe and Planck's scales giving the new light to the regions like black holes, quantum theory, high energy physics, Big Bang theory and cosmology.
\end{abstract}

Index Terms : Relativistic alpha field theory, Determination of field parameters, Electrical field, Gravitational field

\section{INTRODUCTION}

As it is well known, General Relativity Theory (GRT) [1-6] cannot be applied to the extremely strong gravitational field at the Planck's scale, because of the related singularity. Here we present a new theory that is called Relativistic Alpha Field (RAF) theory. We show that RAF theory extends the capability of the GRT for the application to the extremely strong fields at the Planck's scale. This is the consequence of the following predictions of RAF theory: a) no a singularity at the Schwarzschild radius, b) there exists a minimal radius at $r$ $=\left(G M / 2 c^{2}\right)$ that prevents singularity at $r=0$, i.e. the nature protects itself, c) the gravitational force becomes positive (repulsive) if $\left(G M / r c^{2}\right)>1$, that could be a source of a dark energy in the universe, and d) unification of electrical and gravitational forces can be done in the standard four dimensions (4D). Predictions a) and b) are presented in the second part of this theory, while predictions c) and d) are considered in the third part of the theory.

In this (first) part of the theory we present the solution of the field parameters in RAF theory. This solution is based on the assumption that the field parameters should connect geometry of the line element with potential energy of a

Branko Novakovic, FSB - University of Zagreb, Luciceva 5, P.O.B. 509, 10000 Zagreb, Croatia particle in an alpha field given by the related covariant energy equation for a particle with rest mass $m_{0}$. In that sense, the concept of the two dimensionless (unitless) field parameters $\alpha$ and $\alpha^{\prime}$ is introduced. These parameters are scalar functions of the potential energy of a particle in an alpha field. This opened the capability of determination of the field parameters $\alpha$ and $\alpha^{\prime}$ of electrical, gravitational and unified electrical and gravitational fields. The solution of the field parameters is the key point in this theory.

In order to solve the field parameters $\alpha$ and $\alpha^{\prime}$, we started with the derivation of the relative velocity of a particle in an alpha field, $v_{\alpha}$. This relative velocity is derived from the line element in an alpha field given by the nondiagonal form with the Riemannian metrics. Thus, the relative velocity of a particle in an alpha field, $v_{\alpha}$, is described as the function of the field parameters $\alpha$ and $\alpha$ ' and a particle velocity $v$ in the total vacuum (without any potential field). This structure of the relative velocity $v_{\alpha}$ directly connects the line elements of the Special and General Relativity. Namely, in the case of the total vacuum (without any potential field), field parameters $\alpha$ and $\alpha$ ' become equal to unity and, consequently, the relative velocity $v_{\alpha}$ becomes equal to the particle velocity $v$ in the total vacuum. This is the transition of the line element from the General to the Special Relativity.

It is also well known, that for unification of the electroweak and strong interactions with gravity, one can use the following two possibilities [1-6]: a) trying to describe gravity as a gauge theory, or b) trying to describe gauge theories as gravity. The first possibility (a) has attracted a lot of attention, but because of the known difficulties, this approach set gravity apart from the standard gauge theories. The second possibility (b) is much more radical. The initial idea has been proposed by Kaluza-Klein theory [7, 8], which today has many variations [9-14], and takes the place in the modern theories like high energy physics (supergravity [15-17] and string theories [18-29]). These theories use five or more extra dimensions with the related dimensional reduction to the four dimensions. Meanwhile, we do not know the answers to the some questions like: can we take the extra dimensions as a real, or as a mathematical device? In the second [30] and third [31] parts of RAF theory, we show that RAF theory predicts the unification of electrical and gravitational forces in the standard four dimensions (4D). This unification is based on the geometric approach.

RAF theory starts with the main preposition: if the electrical, gravitational and unified fields (forces) can be described by the geometric approach, then the field parameters $\alpha$ and $\alpha^{\prime}$ of a particle in the electrical, gravitational and unified fields should satisfy the Einstein's field equations and the Einstein's geodesic equations. The propositions, related to the satisfaction of the Einstein's field equations and 
the Einstein's geodesic equations are proved in the second [30] and third [31] parts of RAF theory, respectively. In this (first) part of the theory we show the solutions of the field parameters $\alpha$ and $\alpha^{\prime}$ of a particle in the electrical, gravitational and unified fields. If RAF theory is correct, then it could be applied to the both weak and strong fields at the Universe and Planck's scales giving the new light to the regions like black holes, quantum theory, high energy physics, Big Bang theory and cosmology.

This paper is organized as follows. In Sec. II, we show derivation of the relative velocity of a particle in an alpha field $v_{\alpha}$ as the function of the field parameters $\alpha$ and $\alpha^{\prime}$. Derivation of the field parameters $\alpha$ and $\alpha^{\prime}$ in a general form, as the function of the potential energy $U$ is presented in Sec. III. Solutions of the field parameters $\alpha$ and $\alpha^{\prime}$ in electrical and gravitational fields are considered in Sec. IV. The solution of the field parameters $\alpha$ and $\alpha^{\prime}$ in the unified electrical and gravitational field is pointed out in Sec. V. Finally, the related conclusion and the reference list are presented in Sec. VI and Sec. VII, respectively.

\section{DERIVATION OF RELATIVE VELOCITY $V_{\alpha}$}

The basic problem of this paper is to determine the field parameters $\alpha$ and $\alpha^{\prime}$ of a particle in the electrical, gravitational and unified fields. RAF theory is based on the following two definitions:

(a) Definition 1. An alpha field is a potential field that can be described by two scalar dimensionless (unitless) field parameters $\alpha$ and $\alpha^{\prime}$. To this category belong, among the others, electrical and gravitational fields.

(b) Definition 2. Field parameters $\alpha$ and $\alpha^{\prime}$ are described as the scalar dimensionless (unitless) functions of the potential energy $U$ of a particle in an alpha field.

In order to solve the field parameters $\alpha$ and $\alpha^{\prime}$, we started with the derivation of the relative velocity of a particle in an alpha field, $v_{\alpha}$.

(c) Proposition 1. If the line element in an alpha field is defined by the nondiagonal form with the Riemannian metrics [32, 33-35]

$$
\begin{aligned}
d s^{2}= & -\alpha \alpha^{\prime} c^{2} d t^{2}-\kappa\left(\alpha-\alpha^{\prime}\right)_{x} c d t d t-\kappa\left(\alpha-\alpha^{\prime}\right)_{y} c d t d y \\
& -\kappa\left(\alpha-\alpha^{\prime}\right)_{z} c d t d z+d x^{2}+d y^{2}+d z^{2},
\end{aligned}
$$

then the relative velocity of a particle in an alpha field, $v_{\alpha}$, can be described as the function of the field parameters $\alpha$ and $\alpha$ '

$\mathrm{v}_{\alpha}=\mathrm{v}-\frac{\kappa\left(\alpha-\alpha^{\prime}\right) \mathrm{c}}{2}$.

In the previous equation $v$ is a particle velocity in the total vacuum (without any potential field), $c$ is, as usual, the speed of the light in a vacuum and $\kappa$ is a constant determined by the equations (11) and (20).

(d) Proof if the Proposition 1. The line element, given by (1), can be transformed into the new form $d s^{2}=-c^{2} d t^{2}\left(\begin{array}{c}\alpha \alpha^{\prime}+\frac{\kappa\left(\alpha-\alpha^{\prime}\right)_{x} c d x}{c^{2} d t}+\frac{\kappa\left(\alpha-\alpha^{\prime}\right)_{y} c d y}{c^{2} d t} \\ +\frac{\kappa\left(\alpha-\alpha^{\prime}\right)_{z} c d z}{c^{2} d t}-\frac{d x^{2}}{c^{2} d t^{2}}-\frac{d y^{2}}{c^{2} d t^{2}}-\frac{d z^{2}}{c^{2} d t^{2}}\end{array}\right)$

Now, we introduce the following substitutions into (3):

$$
\begin{aligned}
& v_{x}=\frac{d x}{d t}, \quad v_{y}=\frac{d y}{d t}, \quad v_{z}=\frac{d z}{d t}, \quad \rightarrow \\
& v_{x}^{2}+v_{y}^{2}+v_{z}^{2}=v^{2}, \quad \kappa\left(\alpha-\alpha^{\prime}\right)_{x} c v_{x}+\kappa\left(\alpha-\alpha^{\prime}\right)_{y} c v_{y} \\
& +\kappa\left(\alpha-\alpha^{\prime}\right)_{z} c v_{z}=\kappa\left(\alpha-\alpha^{\prime}\right) c v .
\end{aligned}
$$

Applying the substitutions (4) to the relation (3) we obtain the new form of the line element as the function of the particle velocity, $v$, and alpha field parameters $\alpha$ and $\alpha$ '

$$
d s^{2}=-c^{2} d t^{2}\left(\alpha \alpha^{\prime}-\frac{v^{2}}{c^{2}}+\frac{\kappa\left(\alpha-\alpha^{\prime}\right) c v}{c^{2}}\right) \text {. }
$$

The related line element, valid in the Special Relativity, can be obtained from equation (5) by putting $\alpha=\alpha=1$. This is the transition of the line element from the General to the Special Relativity

$d s^{2}=-c^{2} d t^{2}\left(1-\frac{v^{2}}{c^{2}}\right)$

Thus, the form invariant relation of the line element in an alpha field should have the form

$$
d s^{2}=-c^{2} d t^{2}\left(1-\frac{v_{\alpha}^{2}}{c^{2}}\right)
$$

Using the identification between equations (5) and (7) we obtain the following relations:

$$
\begin{array}{r}
\alpha \alpha^{\prime}-\frac{v^{2}}{c^{2}}+\frac{\kappa\left(\alpha-\alpha^{\prime}\right) c v}{c^{2}}=1-\frac{v_{\alpha}^{2}}{c^{2}}, \quad \rightarrow \\
\frac{v_{\alpha}^{2}}{c^{2}}=1-\alpha \alpha^{\prime}+\frac{v^{2}}{c^{2}}-\frac{\kappa\left(\alpha-\alpha^{\prime}\right) c v}{c^{2}} .
\end{array}
$$

Now, we can employ the assumption of the particle velocity, $v_{\alpha}$, given by (2)

$$
\begin{aligned}
v_{\alpha}= & v-\frac{\kappa\left(\alpha-\alpha^{\prime}\right) c}{2}, \rightarrow \\
& \frac{v_{\alpha}^{2}}{c^{2}}=\frac{v^{2}}{c^{2}}-\frac{\kappa\left(\alpha-\alpha^{\prime}\right) c v}{c^{2}}+\frac{\kappa^{2}\left(\alpha-\alpha^{\prime}\right)^{2}}{4} .
\end{aligned}
$$

It follows the comparison between the second relations in (8) and (9) that results by the following identity

$$
\begin{array}{r}
1-\alpha \alpha^{\prime}+\frac{v^{2}}{c^{2}}-\frac{\kappa\left(\alpha-\alpha^{\prime}\right) c v}{c^{2}}=\frac{v^{2}}{c^{2}}-\frac{\kappa\left(\alpha-\alpha^{\prime}\right) c v}{c^{2}} \\
+\frac{\kappa^{2}\left(\alpha-\alpha^{\prime}\right)^{2}}{4}, \rightarrow 1-\alpha \alpha^{\prime}=\frac{\kappa^{2}\left(\alpha-\alpha^{\prime}\right)^{2}}{4} .
\end{array}
$$

The last relation in (10) can be transformed into the simplest form that gives the very important relation between the field parameters $\alpha$ and $\alpha$ ': 


$$
\begin{aligned}
1-\alpha \alpha^{\prime} & =\frac{\kappa^{2}\left(\alpha-\alpha^{\prime}\right)^{2}}{4}, \quad \kappa^{2}=1, \quad \kappa= \pm 1, \\
\rightarrow\left(\frac{\alpha+\alpha^{\prime}}{2}\right)^{2}=1 &
\end{aligned}
$$

From (11) we obtain the definition of the constant $\kappa$ and direct relation between field parameters $\alpha$ and $\alpha$ '. This relation will be employed in the process of the determination of the field parameters $\alpha$ and $\alpha$ '.

Following the previous consideration, we can conclude that the equation (2) describes the relative velocity of a particle in an alpha field $v_{\alpha}$ if the conditions given by (11) are satisfied:

$$
\begin{aligned}
& v_{\alpha}=v-\frac{\kappa\left(\alpha-\alpha^{\prime}\right) c}{2}, \quad \text { if } \kappa^{2}=1, \quad \kappa= \pm 1, \\
& \text { and }\left(\frac{\alpha+\alpha^{\prime}}{2}\right)^{2}=1 .
\end{aligned}
$$

On that way the proof of the proposition 1 is finished.

(e) Proposition 2. The last relation in (12) satisfies the well known condition [1, 2, 33-35] for the metric tensor of the line element (1)

$\sqrt{-\operatorname{det}\left(g_{\mu \nu}\right)}=1$.

(f) Proof of the Proposition 2. The general Riemannian line element [33] can be introduced by the following expression

$$
\begin{aligned}
& d s^{2}=g_{00}\left(d x^{0}\right)^{2}+2 g_{01} d x^{0} d x^{1}+2 g_{02} d x^{0} d x^{2} \\
& +2 g_{03} d x^{0} d x^{3}+g_{11}\left(d x^{1}\right)^{2}+g_{22}\left(d x^{2}\right)^{2}+g_{33}\left(d x^{3}\right)^{2} .
\end{aligned}
$$

Here $g_{\mu \nu}$ are the related metric tensor components of the

Riemannian manifold. Using comparison of the equations (1) and (14), we can conclude that non-null components of the metric tensor $\boldsymbol{g}$ in the line element (1) are determined by the following relations:

$$
\begin{aligned}
& g_{00}=-\alpha \alpha^{\prime}, \quad g_{01}=g_{10}=b_{x}=\frac{-\kappa\left(\alpha-\alpha^{\prime}\right)_{x}}{2}, \\
& g_{02}=g_{20}=b_{y}=\frac{-\kappa\left(\alpha-\alpha^{\prime}\right)_{y}}{2}, \\
& g_{03}=g_{30}=b_{z}=\frac{-\kappa\left(\alpha-\alpha^{\prime}\right)_{z}}{2}, g_{11}=1, g_{22}=1, g_{33}=1 .
\end{aligned}
$$

Following the relations (14) and (15), the general form of the line element (1) can also be presented by the new expression

$$
\begin{aligned}
d s^{2} & =-\alpha \alpha^{\prime}\left(d x^{0}\right)^{2}+2 b_{x} d x^{0} d x^{1}+2 b_{y} d x^{0} d x^{2} \\
& +2 b_{z} d x^{0} d x^{3}+\left(d x^{1}\right)^{2}+\left(d x^{2}\right)^{2}+\left(d x^{3}\right)^{2}
\end{aligned}
$$

Comparing (1) and (16) we can conclude that the related contravariant coordinates of the line elements (1) and (16) are determined by the relations: $d x^{0}=c d t, d x^{1}=d x, d x^{2}=d y, d x^{3}=d z$.

From the relations (15) an (16) one can derive a matrix expression of the components of the general covariant metric tensor $g_{\mu \nu}$ in an alpha field

$$
\left[g_{\mu \nu}\right]=\left[\begin{array}{cccc}
-\alpha \alpha^{\prime} & b_{x} & b_{y} & b_{z} \\
b_{x} & 1 & 0 & 0 \\
b_{y} & 0 & 1 & 0 \\
b_{z} & 0 & 0 & 1
\end{array}\right] .
$$

This metric tensor is symmetric and has ten non-zero elements, as we expected that should be. The matrix expression of the metric tensor (18) is nondiagonal and belongs to the well known Riemannian metrics [33]. Therefore, the related line element (1) is also called a nondiagonal line element. The determinant end the trace of the matrix (18) are presented by the relations:

$$
\begin{gathered}
\operatorname{det}\left[g_{\mu \nu}\right]=-\left(\alpha \alpha^{\prime}+b^{2}\right), \quad T_{R}\left[g_{\mu \nu}\right]=3-\alpha \alpha^{\prime}, \\
b^{2}=b_{x}^{2}+b_{y}^{2}+b_{z}^{2} .
\end{gathered}
$$

Now, we recall the well known condition (13) that should be satisfied by any metric tensor [1,33, 34]. Including the determinant (19) into the condition (13) we obtain the important relation between field parameters $\alpha$ and $\alpha^{\prime}$ :

$$
\begin{aligned}
\sqrt{-\operatorname{det}\left[g_{\mu \nu}\right]}=\sqrt{\alpha \alpha^{\prime}+b^{2}} & =\sqrt{\alpha \alpha^{\prime}+\frac{\kappa^{2}\left(\alpha-\alpha^{\prime}\right)^{2}}{4}}=1, \\
\kappa^{2}=1, & \rightarrow\left(\frac{\alpha+\alpha^{\prime}}{2}\right)^{2}=1 .
\end{aligned}
$$

On that way the proof of the proposition 2 is finished. The condition (20) is also satisfied for $\alpha=\alpha^{\prime}=1$ that is related to the particle motion in a total vacuum (without any potential field). This case belongs to the Special Theory of Relativity.

(g) Proposition 3. Let $\mathrm{d} \tau$ and $\mathrm{dt}$ are differentials of the proper time and coordinate time of the moving particle, respectively. Further, let $H$ is a transformation factor, as an invariant of an alpha field, and $v_{\alpha}$ is a particle velocity in that field given by (2). For that case the transformation factor $H$ has the following form

$$
H=\frac{d t}{d \tau}=\left(1-\frac{v_{\alpha}^{2}}{c^{2}}\right)^{-1 / 2}=\left(\alpha \alpha^{\prime}-\frac{v^{2}}{c^{2}}+\frac{\kappa\left(\alpha-\alpha^{\prime}\right) c v}{c^{2}}\right)^{-1 / 2}
$$

(h) Proof of the Proposition 3. In order to prove the relation in (21) we can start with the usual definition of the differential of proper time $\mathrm{d} \tau$

$d \tau^{2}=\frac{-d s^{2}}{c^{2}}=\frac{1}{H^{2}} d t^{2} \rightarrow H^{2}=\frac{d t^{2}}{d \tau^{2}}=-\frac{1}{d s^{2}} c^{2} d t^{2}$.

Applying the line element (5) to the second relation in (22), we obtain the second form of the transformation factor $H$ given by (21) 
$H=\frac{d t}{d \tau}=\left(\alpha \alpha^{\prime}-\frac{v^{2}}{c^{2}}+\frac{\kappa\left(\alpha-\alpha^{\prime}\right) c v}{c^{2}}\right)^{-1 / 2}$.

On the other hand, employing the identification given in the first relation in (8), one obtains the first form of the transformation factor $\mathrm{H}$ given by (21)

$H=\frac{d t}{d \tau}=\left(1-\frac{v_{\alpha}^{2}}{c^{2}}\right)^{-1 / 2}$.

The first relation in (8) also shows that the first and the second form of the transformation factor $H$ are equal each to the other.

Following the equations (23) and (24) we can conclude that the proposition 3 is proved. Furthermore, if a particle is moving in a total vacuum (without any potential field), then we have $\alpha=\alpha^{\prime}=1$, and the relation (21) is transformed into the transformation factor $\gamma$ valid in the Special Relativity

$$
\begin{gathered}
\alpha=\alpha^{\prime}=1, \rightarrow H=\frac{d t}{d \tau}=\left(1-\frac{v^{2}}{c^{2}}\right)^{-1 / 2}=\gamma=\frac{d t}{d t^{\prime}}, \\
\rightarrow d \tau=d t^{\prime} .
\end{gathered}
$$

\section{SOLUTION OF THE FIELD PARAMETERS}

(i) Proposition 4. Let $m_{0}$ is a rest mass of a particle, $U$ is a potential energy of a particle in an alpha field, $c$ is, as usual, the speed of the light in a vacuum and $(i)$ is an imaginary unit. In that case the field parameters $\alpha$ and $\alpha^{\prime}$ can be described as dimensionless (unitless) functions of the potential energy $U$ of a particle in an alpha field. There are four solutions for both parameters $\alpha$ and $\alpha^{\prime}$ in an alpha field that can be presented by the following relations:

$$
\begin{aligned}
& f(U)=2 U / m_{0} c^{2}+\left(U / m_{0} c^{2}\right)^{2}, \rightarrow \alpha_{1}=1+i \sqrt{f(U)}, \\
& \alpha_{1}^{\prime}=1-i \sqrt{f(U)}, \alpha_{2}=1-i \sqrt{f(U)}, \alpha_{2}^{\prime}=1+i \sqrt{f(U)}, \\
& \alpha_{3}=-1+i \sqrt{f(U)}, \alpha_{3}^{\prime}=-1-i \sqrt{f(U)}, \\
& \alpha_{4}=-1-i \sqrt{f(U)}, \alpha_{4}^{\prime}=-1+i \sqrt{f(U)} .
\end{aligned}
$$

(j) Proof of the Proposition 4. Because there are two field parameters, $\alpha$ and $\alpha^{\prime}$, we have to find out two equations for solution of these parameters. At the first, the field parameters $\alpha$ and $\alpha^{\prime}$ should satisfy the condition given by (20)

$$
\begin{gathered}
\sqrt{-\operatorname{det}\left[g_{\mu \nu}\right]}=\sqrt{\alpha \alpha^{\prime}+\frac{\kappa^{2}\left(\alpha-\alpha^{\prime}\right)^{2}}{4}}=1, \rightarrow \\
\alpha \alpha^{\prime}+\frac{\kappa^{2}\left(\alpha-\alpha^{\prime}\right)^{2}}{4}=1 .
\end{gathered}
$$

From the equation (11) we know that parameter $\kappa= \pm 1$. Thus, we can substitute this identity into the last relation in (27). In that case we obtain the following relation

$$
\alpha \alpha^{\prime}+\frac{\kappa^{2}\left(\alpha-\alpha^{\prime}\right)^{2}}{4}=\alpha \alpha^{\prime}+\frac{\left(\alpha-\alpha^{\prime}\right)^{2}}{4}=\left(\frac{\alpha+\alpha^{\prime}}{2}\right)^{2}=1 \text {. }
$$

Following (27) and (28) we can conclude that the well known condition for the metric tensors given by (13) is transformed into the useful relation between field parameters $\alpha$ and $\alpha^{\prime}$ that should be satisfied

$$
\frac{\alpha+\alpha^{\prime}}{2}= \pm 1, \quad \rightarrow \quad \alpha= \pm 2-\alpha^{\prime} .
$$

Using the multiplication of the last relation in (29) by field parameter $\alpha$ one obtains the following quadratic equation

$$
\alpha^{2} \mp 2 \alpha+\alpha \alpha^{\prime}=0 \text {. }
$$

This is the first equation that will be employed in the process of determination of the field parameters $\alpha$ and $\alpha^{\prime}$.

The second equation should connect the field parameters $\alpha$ and $\alpha^{\prime}$ to the potential energy of a particle in an alpha field. In that sense, we can employ the related covariant energy equation $E_{c}$ for a particle with rest mass $m_{0}$. In order to determine the covariant energy equation $E_{c}$, we can start with the components of the covariant four-momentum vector:

$$
\begin{aligned}
& P_{\mu}=g_{\mu \nu} P^{v}, \quad \mu, v=0,1,2,3, \rightarrow \\
& P_{0}=-\alpha \alpha^{\prime} P^{0}+b_{x} P^{1}+b_{y} P^{2}+b_{z} P^{3}, P_{1}=b_{x} P^{0}+P^{1}, \\
& P_{2}=b_{y} P^{0}+P^{2}, P_{3}=b_{z} P^{0}+P^{3}, \\
& P^{0}=H m_{0} c, P^{1}=H m_{0} v^{1}, P^{2}=H m_{0} v^{2}, P^{3}=H m_{0} v^{3} .
\end{aligned}
$$

Here $P^{v}$ are the components of the contravariant four-momentum vector and $H$ is given by (21). The covariant energy equation $E_{c}$ in an alpha field can be derived by using the following relations:

$$
\begin{aligned}
P_{0}= & \frac{-E_{c}}{c}, \rightarrow E_{c}=-P_{0} c=\alpha \alpha^{\prime} P^{0} c-b_{x} P^{1} c \\
& -b_{y} P^{2} c-b_{z} P^{3} c .
\end{aligned}
$$

Now, we can substitute the contravariant momentums $P^{v}$ from (31) and parameters $\left(b_{x}, b_{y}, b_{z}\right)$ from (15) into (32). As the result we obtain the covariant energy equation $E_{c}$, valid for an alpha field:

$$
\begin{gathered}
E_{c}=H m_{0} \alpha \alpha^{\prime} c^{2}+\frac{H m_{0} \kappa\left(\alpha-\alpha^{\prime}\right) c v}{2}, \\
H=\left(\alpha \alpha^{\prime}-\frac{v^{2}}{c^{2}}+\frac{\kappa\left(\alpha-\alpha^{\prime}\right) c v}{c^{2}}\right)^{-1 / 2} .
\end{gathered}
$$

From (33) we can see that the covariant energy equation $E_{c}$ is in the linear form. The same equation has also been obtained by derivation of the generalized relativistic Hamiltonian, $\boldsymbol{H}_{\alpha}=E_{c}$, in an alpha field [36]. The related nonlinear equation of $E_{c}$ can be obtained by applying of the square operation to the relation (33) (see [36]). This new relation is form invariant and consistent to the related equation in the Special Relativity.

Now we assume that a particle is standing in an alpha field. For that case a particle velocity is equal to zero $(v=0)$ and the relations in (33) are transformed into the following equations:

$$
\begin{gathered}
v=0 \rightarrow E_{c}=H m_{0} \alpha \alpha^{\prime} c^{2}, \quad H=\frac{1}{\sqrt{\alpha \alpha^{\prime}}} \rightarrow \\
E_{c}=m_{0} c^{2} \sqrt{\alpha \alpha^{\prime}} .
\end{gathered}
$$


On the other hand, we know that the energy of the particle with rest mass $m_{0}$ standing in a potential field $(v=0)$ is equal to the sum of the rest mass energy $m_{0} c^{2}$ and the related potential energy $U$ of the particle in that field [37-39]

$$
E_{c}=m_{0} c^{2}+U=m_{0} c^{2}\left(1+\frac{U}{m_{0} c^{2}}\right) \text {. }
$$

Comparing the relations (34) and (35), we can recognize the following identity

$$
\sqrt{\alpha \alpha^{\prime}}=\left(1+\frac{U}{m_{0} c^{2}}\right) \rightarrow \alpha \alpha^{\prime}=\left(1+\frac{U}{m_{0} c^{2}}\right)^{2} .
$$

This is the second equation that will be employed in the process of determination of the field parameters $\alpha$ and $\alpha^{\prime}$. By the inclusion of the second relation in (36) into (30) we obtain the quadratic equation in the following form

$$
\alpha^{2} \mp 2 \alpha+\left(1+\frac{U}{m_{0} c^{2}}\right)^{2}=0 \text {. }
$$

This quadratic equation can be split into the two related quadratic equations:

$$
\alpha^{2}-2 \alpha+\left(1+\frac{U}{m_{0} c^{2}}\right)^{2}=0, \alpha^{2}+2 \alpha+\left(1+\frac{U}{m_{0} c^{2}}\right)^{2}=0 \text {. }
$$

The first quadratic relation in (38) gives the first two solutions of the field parameter $\alpha_{1}$ and $\alpha_{2}$, while the second quadratic relation in (38) gives the next two solutions of the field parameter $\alpha_{3}$ and $\alpha_{4}$ :

$$
\begin{gathered}
\alpha_{1,2}=1 \pm i \sqrt{\frac{2 U}{m_{0} c^{2}}+\left(\frac{U}{m_{0} c^{2}}\right)^{2}}, \\
\alpha_{3,4}=-1 \pm i \sqrt{\frac{2 U}{m_{0} c^{2}}+\left(\frac{U}{m_{0} c^{2}}\right)^{2}} .
\end{gathered}
$$

The related four solutions of the field parameter $\alpha^{\prime}$ can be obtained by the substitution of the parameters $\alpha$ from (39) into the last relation in (29):

$$
\begin{gathered}
\alpha_{1,2}^{\prime}=1 \mp i \sqrt{\frac{2 U}{m_{0} c^{2}}+\left(\frac{U}{m_{0} c^{2}}\right)^{2}}, \\
\alpha_{3,4}^{\prime}=-1 \mp i \sqrt{\frac{2 U}{m_{0} c^{2}}+\left(\frac{U}{m_{0} c^{2}}\right)^{2}} .
\end{gathered}
$$

Thus, the four solutions of the field parameters $\alpha$ and $\alpha^{\prime}$ can be obtained by the unification of the two parameter structures given by (39) and (40):

$$
\begin{aligned}
& f(U)=2 U / m_{0} c^{2}+\left(U / m_{0} c^{2}\right)^{2}, \rightarrow \\
& \alpha_{1,2}=1 \pm i \sqrt{f(U)}, \alpha_{1,2}^{\prime}=1 \mp i \sqrt{f(U)}, \\
& \alpha_{3,4}=-1 \pm i \sqrt{f(U)}, \alpha_{3}^{\prime}=-1 \mp i \sqrt{f(U)} .
\end{aligned}
$$

Because the relations in (41) are equal to the relations in (26), we conclude that the proposition 4 is proved.
Further, it is easy to prove that all $\alpha_{i} \alpha^{\prime}{ }_{i}$ pairs from (41) satisfy the relation (36) giving an invariant $\alpha \alpha^{\prime}$

$\alpha_{i} \alpha_{i}^{\prime}=\left(1+\frac{U}{m_{0} c^{2}}\right)^{2}=\alpha \alpha^{\prime}, \quad i=1,2,3.4$.

For calculation some of the quantities in an alpha field we often need to know the difference of the field parameters $\left(\alpha-\alpha^{\prime}\right)$ :

$\alpha_{1}-\alpha_{1}^{\prime}=2 i \sqrt{f(U)}, \quad \alpha_{2}-\alpha_{2}^{\prime}=-2 i \sqrt{f(U)}$,

$\alpha_{3}-\alpha_{3}^{\prime}=2 i \sqrt{f(U)}, \quad \alpha_{4}-\alpha_{4}^{\prime}=-2 i \sqrt{f(U)}$,

$\left(\alpha_{1}-\alpha_{1}^{\prime}\right)=\left(\alpha_{3}-\alpha_{3}^{\prime}\right), \quad\left(\alpha_{2}-\alpha_{2}^{\prime}\right)=\left(\alpha_{4}-\alpha_{4}^{\prime}\right)$.

The obtained relations in (41), (42) and (43) are valid generally and for their calculation we only need to know potential energy $U$ of the particle in the related potential field

(k)Remarks 1. From the equations (41), (42) and (43) we can see that there are three very important properties of the solutions of the field parameters $\alpha$ and $\alpha^{\prime}$ : a) parameters $\alpha$ and $\alpha^{\prime}$ are dimensionless (unitless) field parameters, b) there are four solutions of the field parameters $\alpha$ and $\alpha^{\prime}$ that reminds us to the Dirac's theory [37] , c) the quantity $\alpha \alpha^{\prime}$ is an invariant related to the four solutions of the field parameters $\alpha$ and $\alpha^{\prime}$.

\section{SOLUTION OF THE FIELD PARAMETERS IN ELECTRICAL AND GRAVITATIONAL FIELDS}

If a particle is an electron that is present in an electrical field, then the potential energy of the electron in that field $U_{e}$ is described by the well known relation [37-39]

$U_{e}=q V=q A_{0}$

Here $q$ is an electric charge of the electron and $V=A_{0}$ is a scalar potential of that field. The four solutions of the field parameters $\alpha$ and $\alpha^{\prime}$ for an electron in an electrical field can be obtained by the substitution of the potential energy $U_{e}$ from (44) into the general relations given by (41):

$$
\begin{aligned}
& f\left(U_{e}\right)=2 q V / m_{0} c^{2}+\left(q V / m_{0} c^{2}\right)^{2}, \rightarrow \\
& \alpha_{1}=1+i \sqrt{f\left(U_{e}\right)}, \alpha_{1}^{\prime}=1-i \sqrt{f\left(U_{e}\right)}, \\
& \alpha_{2}=\alpha_{1}^{\prime}, \quad \alpha_{2}^{\prime}=\alpha_{1}, \quad \alpha_{3}=-1+i \sqrt{f\left(U_{e}\right)}, \\
& \alpha_{3}^{\prime}=-1-i \sqrt{f\left(U_{e}\right)}, \quad \alpha_{4}=\alpha_{3}^{\prime}, \quad \alpha_{4}^{\prime}=\alpha_{3}, \\
& q V<<m_{0} c^{2},\left(q V / m_{0} c^{2}\right)^{2} \cong 0, f\left(U_{e}\right)=2 q V / m_{0} c^{2} .
\end{aligned}
$$

Here $(i)$ is an imaginary unit and $m_{0}$ is a rest mass of the electron. The first four lines in (45) describe a strong electrical field. If the quadric term is close to zero, $\left(q V / m_{0} c^{2}\right)^{2} \approx 0$, then the field parameters (45) describe a weak electrical field. It is easy to prove that the all $\alpha \alpha^{\prime}$ pairs from (45) satisfy the relations in (34), (35) and (36):

$\alpha_{i} \alpha_{i}^{\prime}=\left(1+\frac{q V}{m_{0} c^{2}}\right)^{2}=\alpha \alpha^{\prime}, \quad \sqrt{\alpha \alpha^{\prime}}=\left(1+\frac{q V}{m_{0} c^{2}}\right)$, $v=0, E_{c}=m_{0} c^{2} \sqrt{\alpha \alpha^{\prime}}=m_{0} c^{2}\left(1+\frac{q V}{m_{0} c^{2}}\right)=m_{0} c^{2}+q V$. 
Here $E_{c}$ is the covariant energy of an electron standing $(v=0)$ in an electric field. The differences of the field parameters $\left(\alpha-\alpha^{\prime}\right)$ for an electron in an electrical field have the form:

$$
\begin{aligned}
& \left(\alpha_{1}-\alpha_{1}^{\prime}\right)=\left(\alpha_{3}-\alpha_{3}^{\prime}\right)=2 i \sqrt{\frac{2 q V}{m_{0} c^{2}}+\left(\frac{q V}{m_{0} c^{2}}\right)^{2}}, \\
& \left(\alpha_{2}-\alpha_{2}^{\prime}\right)=\left(\alpha_{4}-\alpha_{4}^{\prime}\right)=-2 i \sqrt{\frac{2 q V}{m_{0} c^{2}}+\left(\frac{q V}{m_{0} c^{2}}\right)^{2}} .
\end{aligned}
$$

(l) Remarks 2. The $\alpha \alpha^{\prime}$ term is a quadratic function of the potential energy of an electron in an electrical field. But the related covariant energy $E_{c}$ of an electron, standing $(v=0)$ in an electrical field, is a linear function of that potential energy. This transformation is obtained here on the natural way, without any a priory assumption. In the second part [30] of this theory it has been shown that field parameters (45) satisfy the Einstein's field equations with a cosmological constant $\Lambda=0$. In the case of a strong static electrical field, the quadratic term $\left(q V / m_{0} c^{2}\right)^{2}$ generates the related energy-momentum tensor $T_{\mu \eta}$ for the static field. For that case we do not need to add by hand the related energy-momentum tensor $T_{\mu \eta}$ of the electrical field on the right side of the Einstein's field equations. In the case of a weak static electrical field, the quadratic term $\left(q V / m_{0} c^{2}\right)^{2} \approx 0$, and the field parameters (45) satisfy the Einstein's field equations in a vacuum $\left(T_{\mu \eta}=0, \Lambda=0\right)$.

If a particle with the rest mass $m_{0}$ is in a gravitational field, then the potential energy of the particle in that field $U_{g}$ is described by the well known relation [1-6]

$U_{g}=m_{0} V_{g}=m_{0} A_{g 0}=-\frac{m_{0} G M}{r}$.

Here $V_{g}=A_{g 0}$ is a scalar potential of the gravitational field, $G$ is the gravitational constant, $M$ is a gravitational mass and $r$ is a gravitational radius. The four solutions of the field parameters $\alpha$ and $\alpha^{\prime}$ for the particle in a gravitational field can be obtained by the substitution of the potential energy $U_{g}$ from (48) into the general relations in (41):

$$
\begin{aligned}
& i \sqrt{f\left(U_{g}\right)}=-\sqrt{2 G M / r c^{2}-\left(G M / r c^{2}\right)^{2}}=-\sqrt{\cdot}, \rightarrow \\
& \alpha_{1}=1-\sqrt{\cdot}, \quad \alpha_{1}^{\prime}=1+\sqrt{\cdot}, \quad \alpha_{2}=\alpha_{1}^{\prime}, \quad \alpha_{2}^{\prime}=\alpha_{1}, \\
& \alpha_{3}=-1-\sqrt{\cdot}, \quad \alpha_{3}^{\prime}=-1+\sqrt{\cdot}, \quad \alpha_{4}=\alpha_{3}^{\prime}, \quad \alpha_{4}^{\prime}=\alpha_{3}, \\
& G M \ll r c^{2}, \rightarrow\left(G M / r c^{2}\right)^{2} \cong 0, \rightarrow i \sqrt{f\left(U_{g}\right)}=-\sqrt{2 G M / r c^{2}} .
\end{aligned}
$$

The first three lines in equations (49) describe a strong gravitational field. If the quadratic term $\left(G M / r c^{2}\right)^{2} \approx 0$ then the field parameters (49) describe a weak gravitational field as we have in our solar system. It is easy to prove that the all $\alpha \alpha^{\prime}$ pairs from (49) satisfy the relations in (34), (35) and (36) for a particle that is standing $(v=0)$ in a gravitational field:

$$
\begin{aligned}
& \sqrt{\alpha \alpha^{\prime}}=\left(1-\frac{G M}{r c^{2}}\right) \rightarrow \alpha \alpha^{\prime}=\left(1-\frac{G M}{r c^{2}}\right)^{2}, \\
& v=0 \rightarrow E_{c}=m_{0} c^{2} \sqrt{\alpha \alpha^{\prime}}=m_{0} c^{2}-\frac{m_{0} G M}{r} .
\end{aligned}
$$

The differences of the field parameters $\left(\alpha-\alpha^{\prime}\right)$ for a particle in a gravitational field have the forms:

$$
\begin{aligned}
& \alpha_{1}-\alpha_{1}^{\prime}=-2 \sqrt{\frac{2 G M}{r c^{2}}-\left(\frac{G M}{r c^{2}}\right)^{2}}, \alpha_{3}-\alpha_{3}^{\prime}=\left(\alpha_{1}-\alpha_{1}^{\prime}\right), \\
& \alpha_{2}-\alpha_{2}^{\prime}=2 \sqrt{\frac{2 G M}{r c^{2}}-\left(\frac{G M}{r c^{2}}\right)^{2}}, \alpha_{4}-\alpha_{4}^{\prime}=\left(\alpha_{2}-\alpha_{2}^{\prime}\right) .
\end{aligned}
$$

(m) Remarks 3. The $\alpha \alpha^{\prime}$ term in (50) is a quadratic function of the potential energy of a particle in a gravitational field. But the related covariant energy $E_{c}$ of a particle standing $(v=0)$ in a gravitational field is a linear function of that potential energy. This transformation has been obtained here on the natural way, without any a priory assumption. In the second part [30] of this theory it has been shown that field parameters (49) satisfy the Einstein's field equations with a cosmological constant $\Lambda=0$. In the case of a strong static gravitational field [40-43,47], the quadratic term $\left(G M / r c^{2}\right)^{2}$ generates the related energy-momentum tensor $T_{\mu \eta}$ for the static field. For that case we do not need to add by hand the related energy-momentum tensor $T_{\mu \eta}$ on the right side of the Einstein's field equations.

The second interpretation could be that the quadratic term $\left(G M / r c^{2}\right)^{2}$ generates the cosmological parameter $\Lambda$ as a function of a gravitational radius [44] for $T_{\mu \eta}=0$. It has been shown [45] that this solution of $\Lambda$ is valid for both Planck's and cosmological scales. In the case of a weak static gravitational field, like in our solar system, the field parameters (49) satisfy the Einstein's field equations in a vacuum $\left(T_{\mu \eta}=0, \Lambda=0\right)$. The general metrics of the relativistic alpha field theory [32] has been applied to the derivation of dynamic model of nanorobot motion in multipotential field [46].

\section{SOLUTION OF THE FIELD PARAMETERS IN UNIFIED ELECTRICAL AND GRAVITATIONAL FIELD}

Let the source of the unified electrical and gravitational fields is an object with mass $\mathrm{M}$, electric point charge $\mathrm{Q}$ and radius $r$. Thus, if a particle is an electron with a rest mass $\mathrm{m}_{0}$ and an electric charge $\mathrm{q}$, then the potential energy of the electron in the unified field, $\mathrm{U}$, is described by the relation [37-39]

$$
U=U_{e}+U_{g}=\frac{q Q}{r}-\frac{m_{0} G M}{r}=q A_{e 0}+m_{0} A_{g 0} .
$$

Here $U_{e}$ is the potential energy of a particle in an electrical field, $U_{g}$ is the potential energy of the particle in a gravitational field, $A_{e 0}$ is a scalar electric potential, $A_{g 0}$ is a scalar gravitational potential and $G$ is a gravitational constant. The potential energy function $f(U)$ for this unified field can be obtained by using the following relations: 


$$
\begin{aligned}
& f(U)=\frac{2 U}{m_{0} c^{2}}+\left(\frac{U}{m_{0} c^{2}}\right)^{2}, \frac{U}{m_{0} c^{2}}=\frac{q Q}{m_{0} r c^{2}}-\frac{m_{0} G M}{m_{0} r c^{2}} \\
& =\frac{G_{e} Q}{r c^{2}}-\frac{G M}{r c^{2}}=\frac{M_{e g}}{r c^{2}}, G_{e}=\frac{q}{m_{0}}, f(U)=\frac{2 M_{e g}}{r c^{2}}+\left(\frac{M_{e g}}{r c^{2}}\right)^{2} .
\end{aligned}
$$

Parameter $G_{e}=q / m_{0}$ is the Kaluza-Klein constant [6-8], obtained here on the natural way. The four solutions of the field parameters $\alpha$ and $\alpha^{\prime}$ for the particle in the unified field can be obtained by the substitution of the potential energy function $f(U)(53)$ into the general relations (41):

$$
\begin{aligned}
& f(U)=2 M_{e g} / r c^{2}+\left(M_{e g} / r c^{2}\right)^{2}, \quad \rightarrow \\
& \alpha_{1}=1+i \sqrt{f(U)}, \quad \alpha_{1}^{\prime}=1-i \sqrt{f(U)}, \\
& \alpha_{2}=\alpha_{1}^{\prime}, \quad \alpha_{2}^{\prime}=\alpha_{1}, \quad \alpha_{3}=-1+i \sqrt{f(U)}, \\
& \alpha_{3}^{\prime}=-1-i \sqrt{f(U)}, \alpha_{4}=\alpha_{3}^{\prime}, \quad \alpha_{4}^{\prime}=\alpha_{3}, \\
& M_{e g} \ll r c^{2}, \rightarrow\left(M_{e g} / r c^{2}\right)^{2} \cong 0, \rightarrow f(U)=2 M_{e g} / r c^{2} .
\end{aligned}
$$

The first three lines in (54) describe a strong unified field. If the quadratic term is close to zero, $\left(M_{e g} / r c^{2}\right)^{2} \approx 0$, then the field parameters (54) describe a weak unified field. It is easy to prove that the all $\alpha \alpha^{\prime}$ pairs in (54) satisfy the relations in (34), (35) and (36):

$$
\begin{aligned}
\alpha_{i} \alpha_{i}^{\prime} & =\left(1+\frac{M_{e g}}{r c^{2}}\right)^{2}=\alpha \alpha^{\prime}, \sqrt{\alpha \alpha^{\prime}}=\left(1+\frac{M_{e g}}{r c^{2}}\right), v=0, \rightarrow \\
E_{c}= & m_{0} c^{2} \sqrt{\alpha \alpha^{\prime}}=m_{0} c^{2}\left(1+\frac{M_{e g}}{r c^{2}}\right)=m_{0} c^{2}+\frac{m_{0} M_{e g}}{r} \\
& =m_{0} c^{2}+\frac{q Q}{r}-\frac{m_{0} G M}{r} .
\end{aligned}
$$

Here $E_{c}$ is the covariant energy of an electron standing $(\mathrm{v}=0)$ in the unified field. The differences of the field parameters $\left(\alpha-\alpha^{\prime}\right)$ for an electron in the unified field have the forms:

$$
\begin{aligned}
& \left(\alpha_{1}-\alpha_{1}^{\prime}\right)=\left(\alpha_{3}-\alpha_{3}^{\prime}\right)=2 i \sqrt{\frac{2 M_{e g}}{r c^{2}}+\left(\frac{M_{e g}}{r c^{2}}\right)^{2}}, \\
& \left(\alpha_{2}-\alpha_{2}^{\prime}\right)=\left(\alpha_{4}-\alpha_{4}^{\prime}\right)=-2 i \sqrt{\frac{2 M_{e g}}{r c^{2}}+\left(\frac{M_{e g}}{r c^{2}}\right)^{2}} .
\end{aligned}
$$

(n)Remarks 4. The $\alpha \alpha^{\prime}$ term is a quadratic function of the potential energy of an electron in the unified field. But the related covariant energy $E_{c}$ of an electron standing $(v=0)$ in the unified field is a linear function of that potential energy. This transformation has been obtained here also on the natural way, without any a priory assumption. In the second part [30] of this theory it has been shown that field parameters (54) satisfy the Einstein's field equations without a cosmological constant $(\Lambda=0)$. In the case of the strong unified field, the quadratic term $\left(M_{e g} / r c^{2}\right)^{2}$ generates the related energy-momentum tensor $T_{\mu \eta}$ of the unified field. In the case of a weak unified field the quadratic term $\left(M_{e g} / r c^{2}\right)^{2} \approx 0$, and the field parameters satisfy the Einstein's field equations in a vacuum $\left(T_{\mu \eta}=0, \Lambda=0\right)$. Following this approach, the unified energy momentum tensor and geodesics equations, with the unified electrical and gravitational forces in 4D, are presented in the second [30] and third [31] parts of this theory, respectively.

\section{CONCLUSION}

In this paper a new Relativistic Alpha Field (RAF) theory is introduced. This theory is based on the concept of the two dimensionless (unitless) field parameters $\alpha$ and $\alpha^{\prime}$. These parameters are scalar functions of the potential energy of a particle in an alpha field. This approach opens the capability of determination of the field parameters $\alpha$ and $\alpha^{\prime}$ for electrical, gravitational and unified electrical and gravitational fields in a standard four dimension (4D). Thus, the solution of the field parameters is the key point in this theory.

Very important predictions of RAF theory are pointed out as follows: a) no a singularity at the Schwarzschild radius, b) there exists a minimal radius at $r=\left(G M / 2 c^{2}\right)$ that prevents singularity at $r=0$, i.e. the nature protects itself, c) the gravitational force becomes positive (repulsive) if $\left(G M / r c^{2}\right)$ > 1 , that could be a source of a dark energy, and d) unification of electrical and gravitational forces can be done in the standard four dimensions (4D). Predictions a) and b) are presented in the second part [30] of this theory, while predictions c) and d) are considered in the third part [31] of the theory. If the predictions of RAF theory are correct, then one can conclude that this theory extends the application of GRT to the extremely strong fields at the Planck's scale. This could give the new light to the regions like black holes, quantum theory, high energy physics, Big Bang theory and cosmology. The next paper will be devoted to the application of RAF theory to control of nanorobot motion in a multipotential field.

\section{ACKNOWLEDGMENTS}

The author wishes to thank to the anonymous reviewers for a variety of helpful comments and suggestions. This work is supported by grants (120-1201842-3048) from the National Scientific Foundation of Republic of Croatia.

\section{REFERENCES}

[1] A. Einstein, Ann. Phys. 49, 769-822 (1916).

[2] A. Einstein, The Meaning of Relativity (Princeton Univ. Press, Princeton, 1955).

[3] C. Sean, Spacetime and Geometry: An introduction to General Relativiry (Amazon.com Books.htm, Hardcover, 2003).

[4] S. Weinberg, Gravitation and Cosmology: Principles and Application of the General Theory of Relativity (Gebundene Ausgabe, RelEspWeinberg.pdf., 1972).

[5] S. W. Hawking, G. F. R. Ellis, The Large Scale Structure of Space-Time (Univ. Press, Cambridge, 1973).

[6] M. Blau, Lecture Notes on General Relativity (A. Einstein Center for Fundamental Physics, Univ. Bern, Bern, 2012, 2014).

[7] T. Kaluza, Zum Unitätsproblem in der Physik (Sitzungsber. Preuss. Akad. Wiss., Berlin, 1921).

[8] O. Klein, Z. Phys. A 37, 895-906 (1926).

[9] E. Witten, Nucl. Phys. B 186, 412-428 (1981).

[10] T. Appelquist, A. Chodos, and P. G. O. Freund, Modern Kaluza-Klein Theories (Addison-Wesley, Menlo Park, Cal., 1987). 
[11] M. J. Duff, Kaluza-Klein Theory in Perspective (Proc. of the Symposium: The Oskar Klein Centenary, World Scientific, Singapore, 22-35, 1994).

[12] J. M. Overduin, and P. S. Wesson, Phys. Rep. 283, 303-378 (1997).

[13] P. S. Wesson, Space-Time-Matter, Modern Kaluza - Klein Theory (World Scientific, Singapore, 1999).

[14] P. S.Wesson, Five-Dimensional Physics: Classical and Quantum Consequences of Kaluza-Klein Cosmology (World Scientific, Singapore, 2006).

[15] D. Z. Freedman, and A. Van Proeyen, Supergravity (Cambridge Univ. Press, Cambridge, 2012)

[16] J. Wess, B. and A. Zumino, Phys. Lett. B 49, 52 (1974)

[17] M. K. Gaillard, and B. Zumino, Nucl. Phys. B 193, 221 (1981).

[18] M. B. Green, J. H. Schwarz, and E. Witten, Superstring Theory (Cambridge Univ. Press, Cambridge, 1987).

[19] J. Polchinski, String Theory (Cambridge Univ. Press, Cambridge, 1998).

[20] R. Brandenberger, and C. Vafa, Nucl. Phys. B 316, 391-410 (1989).

[21] N. Arkani-Hamed, A. G. Cohen, and H. Georgi, Phys. Rev. Lett. 86, 4757 (2001)

[22] C. T. Hill, S. Pokorski, and J. Wang, Phys. Rev. D 64, 105005 (2001).

[23] C. Cshaki, G. D. Kribs, and J. Terning, Phys. Rev. D 65, 015004 (2002)

[24] E. C. Poggio, H. R. Quinn, and S. Weinberg, Phys. Rev. D 13, 1958 (1976).

[25] T. R. Taylor, and G. Veneziano, Phys. Lett. B 212, 147 (1988).

[26] H. C. Cheng, B. A. Dobrescu, and C. T. Hill, Nucl. Phys. B 573, 597 (2000).

[27] C. Cshaki, J. Erlich, C. Grojean, and G. D. Kribs, Phys. Rev. D 65, 015003 (2002)

[28] N. Arkani-Hamed, and M. Schmaltz, Phys. Rev. D 61, 033005 (2000).

[29] M. Gogberashvili, Europhys. Lett. 49, 396 (2000).

[30] B. M. Novakovic, Relativistic alpha field theory - Part II. To be published in IJNTR (2015).

[31] B. M. Novakovic, Relativistic alpha field theory-Part III. To be published in IJNTR (2015).

[32] B. M. Novakovic, Int. J. of Comput. Anticip. Syst., IJCAS 27, p. 93 (2014).

[33] S. Gallot, D. Hullin, and D. J. Lafontane, Riemannian Geometry ( Springer-Verlag, Berlin, New York, ed. 3, 2004).

[34] C. T. J. Dodson, and T. Poston, Tensor Geometry. Graduate Texts in Mathematics (Springer-Verlag, Berlin, New York, ed. 2, 1991), p. 130

[35] M. T. Vaughin, Introduction to Mathematical Physics (Wiley-VCH Verlag GmbH \& Co., Weinheim, 2007).

[36] B. M. Novakovic, in Proceedings of the Ninth Int. Conf. on Comp. Anticip. Syst., Liege, 2009, edited by D. Dubois (University of Liege, Liege 2009), AIP-CP 1303, p. 141 (2010). DOI: 10.1063/1.3527149.

[37] P. A. M. Dirac, Directions in Physics (Wiley, New York, 1978).

[38] I. Supek, Theoretical Physics and Structure of Matter, Part I (Skolska knjiga, Zagreb, 1992).

[39] I. Supek, Theoretical Physics and Structure of Matter, Part II (Skolska knjiga, Zagreb, 1990).

[40] D. H. Perkins, Introduction to High Energy Physics (Cambridge Univ. Press, Cambridge, 2000).

[41] D. Sherman, et al., Nat. Phys. 11, 1-5 (2015).

[42] J. Steinhauer, Nat. Phys. 10, 864-869 (2014).

[43] M. Meckel, et al. Nat. Phys. 10, 594 - 600 (2014)

[44] B. M. Novakovic, D. Novakovic, and A. Novakovic. in Proceedings of the Sixth Int. Conf. on Comp. Anticip. Syst., Liege, 2003, edited by D. Dubois (University of Liege, Liege 2003), AIP-CP 718, p.133 (2004). DOI: $10.1063 / 1.1787318$

[45] B. M. Novakovic, D. Novakovic, and A. Novakovic, in Proceedings of the Seventh Int. Conf. on Comp. Anticip. Syst., Liege, 2005, edited by D. Dubois (University of Liege, Liege 2005), AIP-CP 839, p.144, (2006). DOI: 10.1063/1.2216624.

[46] B. M. Novakovic, Strojarstvo 53 (2), 103-111 (2011).

[47] R. Ding, et al. Phys. Rev. D 92, (015008) (2015).

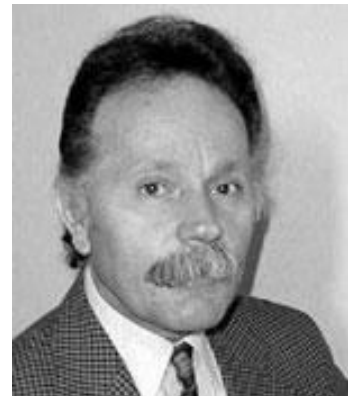

Branko Novakovic is a Professor emeritus at FSB - University of Zagreb, Croatia. Prof. Novakovic received his $\mathrm{PhD}$ from the University of Zagreb in 1978. His research of interest includes physics, control systems, robotics, neural networks, and fuzzy control. He is author of two books, Control Systems (1985) and Control Methods in Robotics, Flexible Manufacturing Systems and Processes (1990), and co-author of a book Artificial Neural Networks (1998). He has published over 220 research papers in his research of interest. 